\title{
A Case Study of an Effective and Sustainable Antiretroviral Therapy Program in Rural South Africa
}

\author{
Karl W. Le Roux, MB, ChB, ${ }^{1,2}$ Emily C. Davis, BA, ${ }^{3}$ Charles Benjamin Gaunt, MB, ChB, ${ }^{1}$ \\ Catherine Young, MA, ${ }^{4}$ Maryann Koussa, $\mathrm{MPH},{ }^{3}$ Carl Harris, $\mathrm{MD},{ }^{5}$ \\ and Mary Jane Rotheram-Borus, $\mathrm{PhD}^{3}$
}

\begin{abstract}
The delivery of high-quality HIV care in rural settings is a global challenge. Despite the successful expansion of antiretroviral therapy (ART) in Africa, viral load (VL) monitoring and ART adherence are poor, especially in rural communities. This article describes a case study of an ART program in the deeply rural Eastern Cape of South Africa. The Zithulele ART Program initiated five innovations over time: (1) establishing district hospital as the logistical hub for all ART care in a rural district, (2) primary care clinic delivery of prepackaged ART and chronic medications for people living with HIV (PLH), (3) establishing central record keeping, (4) incentivizing VL monitoring, and (5) providing hospital-based outpatient care for complex cases. Using a pharmacy database, on-time VL monitoring and viral suppression were evaluated for $882 \mathrm{PLH}$ initiating ART in the Zithulele catchment area in 2013. Among PLH initiating ART, 12.5\% $(n=110)$ were lost to follow-up, 7.7\% $(n=68)$ transferred out of the region, $10.2 \%(n=90)$ left the program and came back at a later date, and $4.0 \%(n=35)$ died. Of the on-treatment population, $82.9 \%(n=480 / 579)$ had VL testing within 7 months and 92.6\% $(n=536 /$ $579)$ by 1 year. Viral suppression was achieved in $85.2 \%$ of those tested $(n=457 / 536)$, or $78.9 \%(n=457 / 579)$ overall. The program's VL testing and suppression rates appear about twice as high as national data and data from other rural centers in South Africa, despite fewer resources than other programs. Simple system innovations can ensure high rates of VL testing and suppression, even in rural health facilities.
\end{abstract}

Keywords: HIV, antiretroviral therapy, rural health care, South Africa, viral suppression, viral load monitoring, prepackaging ART

\section{Introduction}

$\mathbf{I}$

NEQUALITIES IN THE quality and accessibility of health care between urban centers and rural areas is particularly stark in low- and middle-income countries. ${ }^{1}$ Providing highquality health care in rural settings is a global challenge. ${ }^{2}$ For people living in rural areas, large distances, geographical barriers, poor infrastructure, and the high cost of transport affect routine medication access, patient utilization of care, and the level and capacity of health care workers at clinics and hospitals. ${ }^{3,4}$ These barriers to health care are particularly relevant for people living with HIV (PLH) in rural areas who require lifelong antiretroviral therapy (ART) and regular follow-up assessments.
South Africa has 7.2 million PLH, the country with the most PLH globally. ${ }^{5}$ From 2008 to 2017, the proportion of PLH receiving ART has increased from $16.6 \%$ to $61 \%$, reaching an additional three million people, with concurrent drops in HIV mortality. ${ }^{5,6}$ Despite these successes, many challenges remain for the successful provision of ART. After initiating ART, an estimated one-third of PLH in sub-Saharan Africa are lost to follow-up (LTF) after 3 years. ${ }^{7}$ Further, country data reported to UNAIDS shows that only $44 \%$ of PLH on ART receive routine viral load (VL) testing ${ }^{8}$ and only $47 \%$ of PLH in South Africa are known to be virally suppressed. ${ }^{5}$

Statistics on HIV treatment services are difficult to find for rural areas; however, there is a clear gap between rural and

\footnotetext{
${ }^{1}$ Zithulele Hospital, Mqanduli District, South Africa.

${ }^{2}$ Woodrow Wilson School of Public International Affairs at Princeton University, Princeton, New Jersey.

${ }^{3}$ Department of Psychiatry, University of California Los Angeles, Los Angeles, California.

${ }^{4}$ Jabulani Rural Health Foundation, Mqanduli District, South Africa.

${ }^{5}$ Dalens Hospital, Enskededalen, Sweden.
} 
urban South Africans. A recent study found that PLH in rural areas tend to be sicker upon initiation of ART, have VL testing less frequently, and are more likely to have drug resistance. $^{9}$ Nonadherence to treatment and LTF increases the risk of developing AIDS in PLH, the risk of HIV transmission, and the prevalence of resistant strains of HIV nationally. ${ }^{10,11}$

In rural areas, there are a number of predictable challenges. There are almost always too few trained personnel to care for the households in their catchment areas. ${ }^{12}$ District hospitals (DHs) and their associated primary care clinics often do not function as an integrated system, but are instead managed and administrated in parallel. ${ }^{13}$ Clinics typically have fewer less well-trained staff compared with hospitals, and clinic nurses in charge of HIV care often do not receive the necessary training or support to manage complex ART regimens. ${ }^{14-16}$ Patients, particularly those who regularly collect medications for chronic diseases, face major barriers to accessing care, such as significant costs and travel time to go to and from the clinic. ${ }^{17,18}$ For South Africa to improve treatment and monitoring rates and meet the UNAIDS global goals of 90-90-90 by 2030 , the gap between treatment in urban areas and the $43.2 \%$ of the population of rural areas must be addressed with sustainable and high-quality ART programs. ${ }^{19}$ This article provides a case example to meet these challenges.

\section{Methods}

The study was conducted with approval of the Institutional Review Boards of the University of California, Los Angeles (UCLA; IRB No. 16-001362) and Stellenbosch University (No. N12/08/046).

\section{Setting}

This study reports on innovations made in ART provision in the rural King Sabata Dalindyebo subdistrict of the O.R. Tambo district in the Eastern Cape of South Africa. Situated in one of the poorest municipalities in South Africa, Zithulele Hospital is a 146-bed DH where services evolved continuously for the past 13 years. ${ }^{20}$ It serves 11 health clinics and a catchment population of 122,500 people spread out over roughly $1100 \mathrm{~km}^{2}$, as shown in Table 1 . Only 9\% of the population in the catchment area is employed and the average household income is R14,600 each year (about $\$ 1080$ USD). ${ }^{21}$ Only $6 \%$ of the population has water provided by local or regional services, and $34 \%$ do not have access to either a toilet or a latrine. ${ }^{21}$

Table 1. Comparative Descriptions of the Community Context of the Staffing

PatTern in the Zithulele Hospital Network and at the Hlabisa ANTIRETroviral Therapy Program

\begin{tabular}{lcc}
\hline & Zithulele program & Hlabisa program \\
\hline Area, $\mathrm{km}^{2}$ & 1100 & 1430 \\
Population & 122,500 & 228,000 \\
PLH & 4063 & 7576 \\
Peer counselors & 11 & 540 \\
Staff & 7 & 123 \\
\hline
\end{tabular}

PLH, people living with HIV.

\section{Zithulele Hospital innovations in ART management and care}

Between 2006 and 2009, the staff at Zithulele Hospital sequentially implemented a series of innovations that shifted standard operating practice. Each step was accompanied by a strategy to monitor the consistency and quality of the implementation of the innovation. As the program has grown, the five innovations were as follows:

(1) The DH provides logistical and clinical support weekly. Under the organizing principle that the DH is the hub of HIV care for all PLH in the region, the hospital and its 11 closest clinics formed a collaborative network. Hospital doctors routinely rotate through the primary care clinics and develop positive relationships and trust between hospital and clinic staff. Peer counselors who provide support to PLH are trained at the hospital and have access to physicians while operating at all clinics 1 day a week.

(2) Medication prepackaging. A centralized hospital-based team prepackages both HIV-related and all other chronic medications (such as hypertensive, diabetic, and TB medications) needed by PLH in bar-coded bags that provide up to 3 months of treatment. The rationale for prepackaging is that stock-outs of drugs are common. ${ }^{4,22}$ For example, in 2013, 53\% of clinics and hospitals in the region had stock-outs of ART and TB medication, lasting on average 45 days. ${ }^{23}$ Pharmacists at the DH, more skilled and more consistently available than at clinics, secured a continuous supply of ART.

(3) Linking HIV records, medication, and clinical care. In addition to the standard adult clinical record, PLH receive HIV summary cards and HIV dispensing cards (available on request). The summary cards are stapled into patient-held clinic records and contain tabulated blood results over time, all ART regimen changes, a summary of major clinical issues, and follow-up dates for patient reference. The dispensing cards are kept centrally at the DH, but travel to the clinics (packed with medications) to make sure that prescribing information, laboratory data, and critical clinical notes are immediately available for all HIV visits. The administrative team at the hospital is responsible for accurately recording every clinic visit, updating the pharmacy record with new prescriptions, recording all laboratory results on the HIV dispensing cards, and contacting all PLH who have defaulted. A log system routinely monitors adherence to the protocol.

(4) Incentivizing on-time testing with reduced clinic visits. HIV dispensing cards trigger tests such as VL, CD4, and creatinine at assessments, and the months of medication dispensed ensures that PLH return on time for these services. Figure 1 outlines the process for streamlining compliant PLH while bringing others back to clinic more often. For example, if a family member collects medication for a PLH who needs blood drawn, only 1 month of treatment is dispensed until the PLH comes in. Once the results have been reviewed (and the PLH is considered healthy by clinic staff), 3 months of ART is typically dispensed. 


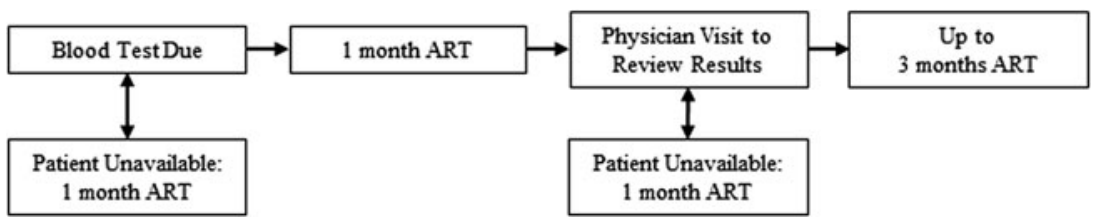

FIG. 1. Flow of clinic visits for PLH to encourage on-time viral load testing and follow-up. PLH, people living with HIV.
(5) Hospital outpatient care for critical patients. The sickest 8-10\% of PLH, adults and children, are retained by the hospital for outpatient clinic care. Although many PLH easily achieve viral suppression with first-line medications, some are sicker before they start ART, have adverse reactions to certain medications, or have strains of HIV resistant to first and second-line regimens. These cases require complex care and are likely to experience repeated crises. Almost all pediatric cases are considered complex or critical patients. For such PLH, the appropriate level of care is at the $\mathrm{DH}$, which offers immediate resources such as $\mathrm{X}$ rays and blood tests and oversight by an experienced physician.

\section{Data collection}

This retrospective cohort was obtained through the Intelligent Dispensing of Antiretroviral Treatment (iDART) software supplied by Cell-Life and used by the hospital to manage the supply of ART medication. All patient data were de-identified for the purpose of this study.

\section{Inclusion criteria}

All PLH who started on ART at Zithulele Hospital or its 11 associated clinics from July 1, 2013 to June 30, 2014 were identified. Although the program was started in 2009, it was only expanded to all 11 primary care clinics by the end of 2011, and we waited to evaluate the efficacy of the innovations until all were clearly implemented with ongoing supervision and efficient procedures. Prematurely evaluating a newly implemented program does not indicate whether the program would be effective or sustainable in the long term, and is likely to evaluate a low-quality program. In addition, 180 patient records with an ART start date in 2013 that did not have classification as being new, a transfer, or a restart due to technical difficulties upgrading the iDART system, were included. These PLH were re-entered within 4 months of the ART start date, and this was not believed to be a significant interruption in the records.

\section{Analysis}

For the entire cohort, PLH description of gender and age of entry into ART as well of the number of transfers out, LFT, and deaths were reported. VL analysis was performed on PLH initiating ART that remained in the Zithulele ART Program for the full year.

"On-time", VL status was defined as a test given between 60 and 210 days after starting ART. These cutoff values were used to avoid inclusion of "baseline" VL tests, while capturing all PLH who were closely followed up within the time period indicated by South African national guidelines. ${ }^{24}$
In addition to "on-time" VL, we looked at PLH with "any" VL, taken between 60 and 365 days after the ART initiation date.

Viral suppression was based on the latest VL results in the 12 months of treatment. A VL of $\leq 50$ copies $/ \mathrm{mL}$ was used as the cutoff for viral suppression as this was the reference value used by our laboratory; adequate suppression was defined as $\leq 400$ copies/mL, which is the threshold for viral suppression used by the South African National Department of Health. ${ }^{25}$ In keeping with other reports, virological failure was defined as latest VL $\geq 1000$ copies/mL, rather than two VL $\geq 10003$ months apart as required for clinical virological failure. ${ }^{24}$

\section{Results \\ Implementation}

In 2006, Zithulele Hospital was the primary center for delivering HIV care in the region. By March 2014, 90.5\% of the 4063 PLH on ART had their HIV care provided at their local clinic (and $92 \%$ by 2017). The scale up of the Zithulele ART Program was achieved with the addition of 11 ART peer counselors, 1 ART manager, 2 pharmacy helpers, 2 administrative staff, 1 data capturer, and 1 default tracer as shown in Table 1. During the 1 year study period, there were $882 \mathrm{PLH}$ who started ART. The majority of PLH who initiated ART in this period were women $(n=597,67.7 \%)$, and the average age of ART initiation was 32 years (standard deviation= 13.4). There were no statistically significant differences based on gender or age of PLH that initiated ART compared with those retained for 1 year.

\section{Outcomes}

After 12 months of treatment, $579(65.6 \%)$ of PLH initially started on ART remained in the program without interruption of care. Of PLH that left the program, 35 (4.0\%) had died, 111 $(12.6 \%)$ transferred out of the area at some point and 43 $(4.9 \%)$ of these later returned, $47(5.3 \%)$ were at first LTF but then returned to care, and $110(12.5 \%)$ were LTF and did not return to ART in the Zithulele catchment area. Figure 2 shows the patients initiating ART and those who left the Zithulele program.

Figure 3 has a breakdown of VL testing and outcomes for PLH remaining on ART. Of PLH who remained on ART within Zithulele Hospital or the 11 clinics in its network, 480 $(82.9 \%)$ completed VL testing "on time" (between 60 and 210 days of ART initiation) and $536(92.6 \%)$ had at least 1 VL at "any" time (between 60 and 365 days of ART initiation). The remaining $43(7.4 \%)$ PLH on ART at Zithulele did not have a recorded VL between 60 and 365 days of treatment. Of the 579 PLH retained in care, 457 (78.9\%) had complete viral suppression, $502(86.7 \%)$ had adequate suppression, and only 32 PLH $(5.5 \%)$ had a VL $\geq 1000$. 


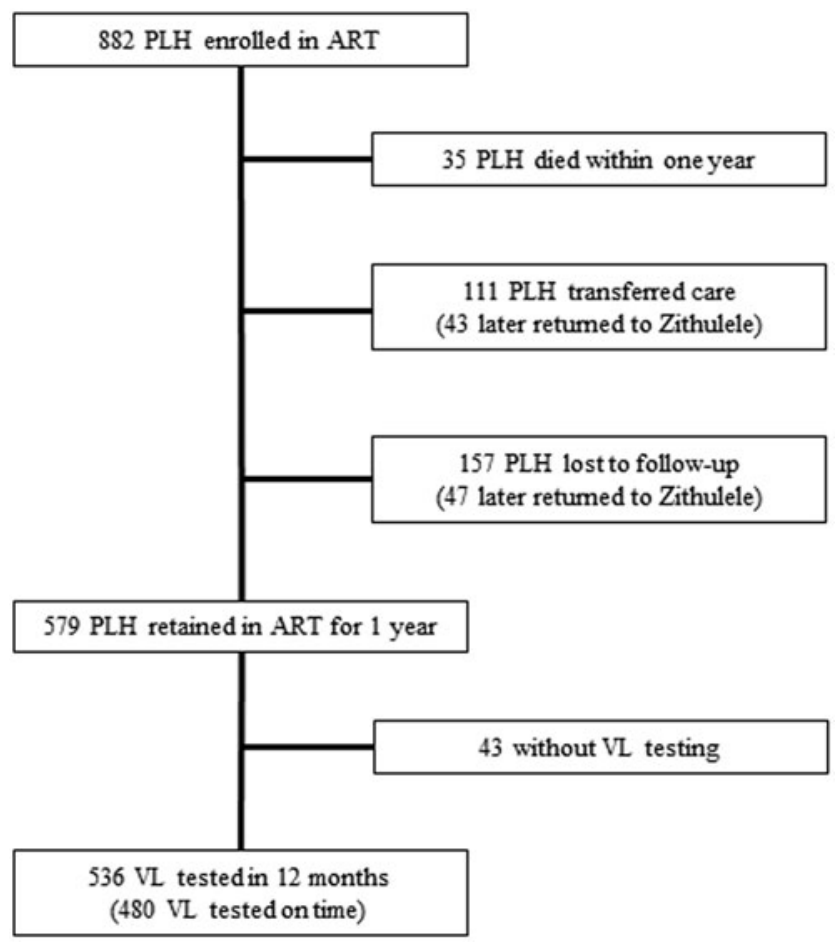

FIG. 2. Study flow and outcomes for PLH initiating ART at Zithulele Hospital or one of the 11 regional clinics in 2013. ART, antiretroviral therapy; PLH, people living with HIV.

\section{Discussion}

Achieving viral suppression prevents transmission of HIV, as well as extending the length and quality of life of PLH. ${ }^{10}$ However, many rural districts struggle to monitor treatment outcomes in PLH on ART, or even maintain adequate records. ${ }^{26}$ This case study describes the protocols used in a successful and sustainable ART program in one of the country's poorest rural districts with significant physical and institutional barriers to accessing health care and offers suggestions for potential means of overcoming those barriers. Many previous attempts in South Africa have been unsuccessful in increasing ART program retention (e.g., economic incentives $)^{27}$ or show promise (e.g., adherence clubs), but lack robust evidence and/or fail to address the structural challenges of rural health care provision. ${ }^{28,29}$

Many of the innovations in the Zithulele ART Program are targeted toward making the system more efficient. Individually, these innovations have been used in other sites. ${ }^{30}$ However, almost all tested clinical innovations are evaluated alone, answering the question: Does this innovation improve the targeted outcome? The benefits only occur when integrated and consistently applied within a health system of a hospital and associated clinics. Only then are the results robust and broadly implemented. Clinics and hospitals in rural areas are often understaffed, making time with a nurse or physician extremely valuable. The use of clinical staff for simple administrative tasks should, therefore, be minimized to allow more time for patient contact and clinical decisionmaking. Further, significant administrative burdens on clinicians can lead to poor record keeping. Research has shown that data on PLH in the Eastern Cape can be highly inaccurate. ${ }^{26}$ Having prepackaged medication, laboratory results, and follow-up dates at hand when seeing PLH allows for more effective patient-clinician interactions. Similarly, keeping critical cases at the hospital ART clinic until they can be safely down-referred frees up clinic nurses to manage routine PLH.

For PLH, each visit to a clinic can be a lengthy and costly experience. Many PLH default on medication when they cannot reach the clinic due to the high cost of taking a taxi or an inability to be away from home for the entire day. ${ }^{18}$ Dispensing 3 months of treatment at time provides a strong incentive for PLH to have their blood tests taken on time and return for the follow-up of results. Patients who miss their VL test dates only get 1 month of treatment dispensed at a time, until their results have been reviewed. Each of these measures engenders trust in the system, decongests clinics on ART days, and can save PLH from making additional lengthy and expensive trips to the clinic or hospital.
FIG. 3. Viral load testing and suppression in a rural on-treatment population.

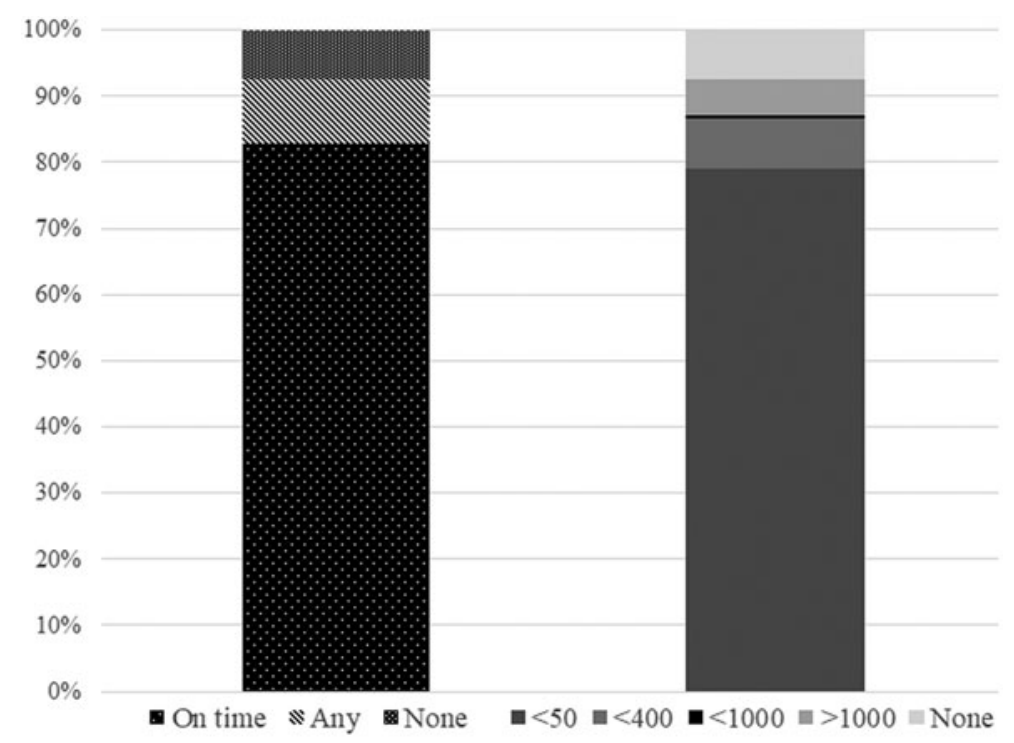


There are potential drawbacks to the system implemented in Zithulele. Medication packages and HIV dispensing cards are kept at the hospital before designated HIV clinic days and only remain with the clinic for 2 weeks to accommodate PLH who miss their assigned return date. Although clinics are able to obtain clinical information from the summary card, can still dispense ART any time, and retain a list of patients who require blood draws, PLH that arrive at the clinic too early, too late, or for any unrelated health problem will not have their medication package as readily available and may not be able to consult with a physician. In addition, PLH that have difficulty getting to a clinic may be frustrated by receiving 1 -month medication packages as a result of missing a required blood test or physician visit. Although we believe that this is a good way to encourage compliance with these national protocols, it may result in some PLH defaulting or even dropping out of care.

Still, the Zithulele ART Program markedly outperforms the country as a whole, with fewer deaths, greater retention in care, and more than double the proportion of its PLH having a recent $\mathrm{VL}$, compared with national figures reported by the South African Department of Health for 2013-2014. ${ }^{31}$ Figure 4 gives a visual representation of Zithulele outcomes, Hlabisa outcomes, and SA DoH figures April 2013 to March 2014. As most other research on successful ART programs comes from either urban areas or dedicated research sites, it is difficult to find a similar comparison with hospitals in rural South Africa. Those that exist are small studies of a hospital PLH base,${ }^{32}$ evaluations of programs that were unsustainable in the long term, ${ }^{33}$ or from a large research site with increased resources and funding. ${ }^{34}$

Hlabisa is relatively good site to compare Zithulele as shown in Table 1. Hlabisa is a rural research site with international funding and a staff that has expanded dramatically when ART provision was expanded. The Hlabisa study described earlier (which newly started ART patients between
2004 and 2008) serves 16 clinics, a catchment of 228,000, and $7576 \mathrm{PLH}$, a little more than twice the size of the $\mathrm{Zi}$ thulele program, but covering an area only $21 \%$ larger (1000 vs. $1400 \mathrm{~km}^{2}$ ). Hlabisa utilized substantial international funding that included 123 PEPFAR-sponsored staff and 540 home-based volunteers, ${ }^{34,35}$ a staffing ratio 40 times that of the Zithulele network. This staffing pattern would not be possible outside of a research context. Yet only $30 \%$ of persons living with HIV had received VL testing at 14 months and only $23.1 \%$ of PLH on treatment were confirmed to be virally suppressed $(<25$ copies $/ \mathrm{mL}) .{ }^{35}$ In contrast, $92 \%$ of PLH receiving ART in the Zithulele Hospital catchment area had their first VL within 1 year of the initiation ART and $79 \%$ of the on-treatment population were confirmed to be virally suppressed $(<50$ copies $/ \mathrm{mL})$ figures that are without precedent in the current literature. The results seen in the Zithulele ART Program appear to reflect the results of iterative improvement in the quality of care over time and use of the resources at hand.

The Zithulele ART Program is challenged by PLH LTF. However, many PLH classified as LTF (defaulting treatment for 3 months in a row) later return with limited or no interruption of treatment, having simply transferred to another facility, or obtained medication in other ways. ${ }^{36}$ The true rate of LTF is difficult to assess in this highly migratory population and without a national database for patient tracking.

\section{Limitations}

The Zithulele ART Program was initiated to make highquality ART available to the catchment area and was not established for research purposes. As a result, data taken from a pharmaceutical dispensing database do not include reasons for missing VL tests. In addition to the tests not being performed, it is possible that the results were not entered, entered incorrectly, or lost. However, these examples reflect

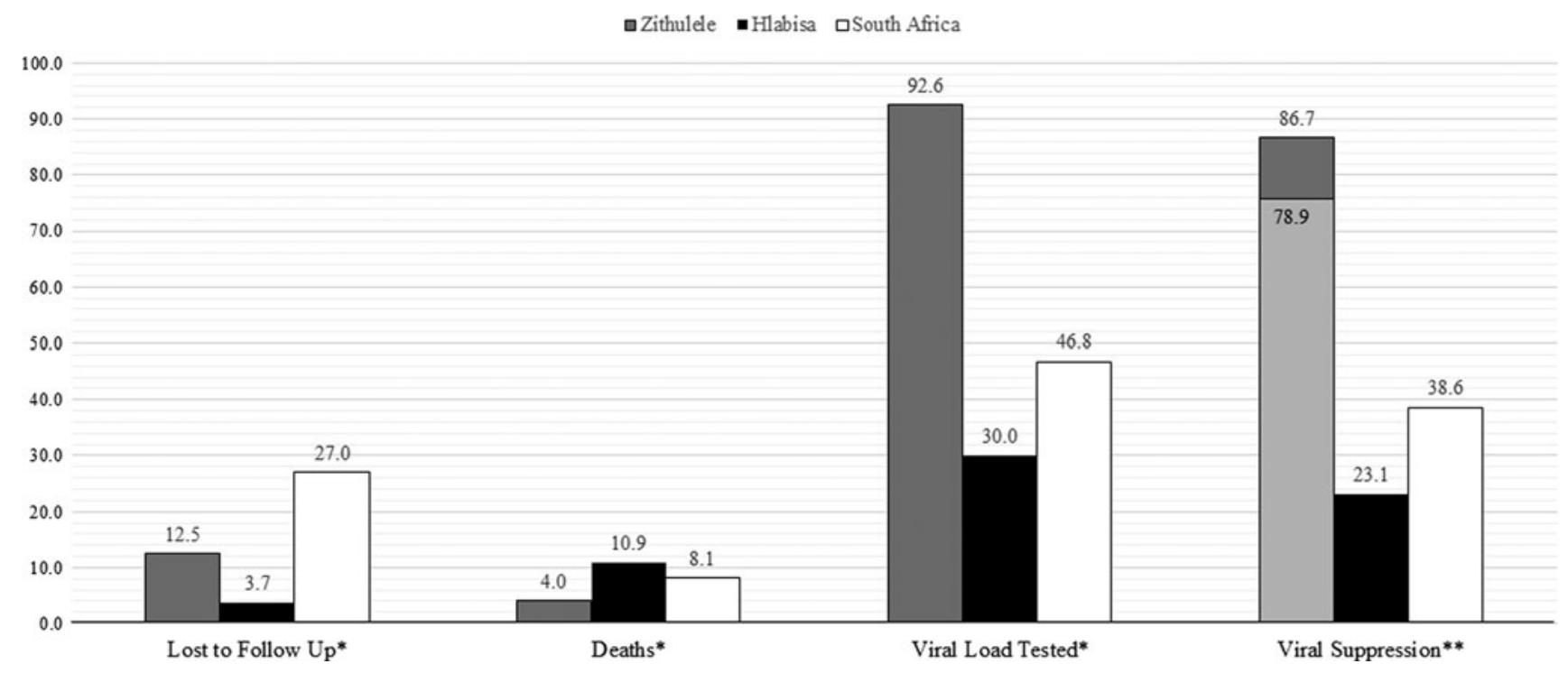

FIG. 4. Zithulele 12-month outcomes compared with Hlabisa and South Africa. *Within the first year of ART treatment. Viral load testing is on the on-treatment population. **Viral suppression is defined as $<400$ dark gray and $<50$ light gray for Zithulele; $<400$ for South African National Data, and $<25$ for Hlabisa. Percentages are taken from the on-treatment population in all three reports. ART, antiretroviral therapy. 
challenges generally faced in government clinics in South Africa and do not significantly impact the validity of the data.

In addition, the Eastern Cape is home to a migratory population that often moves back and forth between regions. The on-treatment inclusion criteria for this study skew the sample toward the more stable two-thirds of the PLH population and may have contributed to positive results. However, this constraint is necessary to evaluate the Zithulele program without including PLH that were seen primarily outside the catchment area. Other articles that evaluate VL status have used an on-treatment population as well. ${ }^{33,35}$

Expanding ART coverage in South Africa is an important goal for reducing the burden of HIV/AIDS. However, expanding ART coverage without a high-quality health care delivery model that ensures that VL testing occurs, results are checked, viral suppression is achieved, and individuals are retained in care is not an effective or sustainable means of combatting HIV/AIDS. In rural areas of South Africa, where there are many barriers to the delivery of high-quality health care, interventions such as the one seen at Zithulele are important in helping reduce the incidence, morbidity, and mortality of HIV. These results demonstrate that it is possible for a rural region to significantly outperform the country as a whole in VL testing and suppression through the systematic implementation of innovations designed to increase clinic efficiency and reduce barriers to accessing care. It is an example of a best practice model that is replicable in other rural settings, and might also be implemented successfully in urban and peri-urban areas.

\section{Acknowledgments}

We thank Cell-Life for providing the iDART software used for record keeping and pharmacy dispensing. The $\mathrm{Zi}$ thulele ART Program would not be possible without the support of the Donald Woods Foundation and the Jabulani Rural Health Foundation.

\section{Authors' Contributions}

K.W.L.R. and C.H. initiated the study. K.W.L.R., C.Y., and C.B.G. made substantial contributions to the Zithulele ART Program. All authors contributed to the design of the work. E.C.D., C.Y., and C.H. contributed to the acquisition of data. E.C.D. analyzed the data. K.W.L.R., E.C.D., C.Y., C.H., and M.J.R.-B. contributed to the interpretation of data. K.W.L.R., E.C.D., C.H., and M.K. contributed to drafting the document. K.W.L.R., E.C.D., C.B.G., C.Y., and M.J.R.-B. provided critical revisions to the document.

\section{Author Disclosure Statement}

The authors of this study have no financial relationships to disclose that could inappropriately influence or bias their work.

\section{Funding Information}

This study is supported by the National Institute of Mental Health (R01MH111391). No outside funders played any role in the study design, in the collection, analysis, and interpretation of data, in the writing of the report, or in the decision to submit the article for publication.

\section{References}

1. Strasser R, Kam SM, Regalado SM. Rural health care access and policy in developing countries. Annu Rev Public Health 2016;37:395-412.

2. Weinhold I, Gurtner S. Understanding shortages of sufficient health care in rural areas. Health Policy 2014;118:201-214.

3. Harris B, Goudge J, Ataguba JE, et al. Inequities in access to health care in South Africa. J Public Health Policy 2011; 32(Suppl 1):S102-S123.

4. Macha J, Harris B, Garshong B, et al. Factors influencing the burden of health care financing and the distribution of health care benefits in Ghana, Tanzania and South Africa. Health Policy Plan 2012;27(Suppl 1):i46-i54.

5. Joint United Nations Programme on HIV/AIDS (UNAIDS). UNAIDS Data 2018. 2018. Available at: www.unaids.org/ sites/default/files/media_asset/unaids-data-2018_en.pdf (Last accessed March 15, 2019).

6. Joint United Nations Programme on HIV/AIDS (UNAIDS). South Africa HIV Epidemic Profile. 2014. Available at: http://docplayer.net/34533779-Unaids-2014-south-africahiv-epidemic-profile.html (Last accessed March 11, 2019).

7. Haas AD, Zaniewski E, Anderegg N, et al. Retention and mortality on antiretroviral therapy in sub-Saharan Africa: Collaborative analyses of HIV treatment programmes. J Int AIDS Soc 2018;21:e25084.

8. Joint United Nations Programme on HIV/AIDS (UNAIDS). UNAIDS Data 2017. 2017. Available at: www.unaids.org/ sites/default/files/media_asset/Global_AIDS_update_2017_ en.pdf (Last accessed March 15, 2019).

9. Rossouw TM, Nieuwoudt M, Manasa J, et al. HIV drug resistance levels in adults failing first-line antiretroviral therapy in an urban and a rural setting in South Africa. HIV Med 2017;18:104-114.

10. Tanser F, Barnighausen T, Grapsa E, et al. High coverage of ART associated with decline in risk of HIV acquisition in rural KwaZulu-Natal, South Africa. Science 2013;339: 966-971.

11. Schaecher KL. The importance of treatment adherence in HIV. Am J Manag Care 2013;19(12 Suppl):s231-s237.

12. Mburu G, George G. Determining the efficacy of national strategies aimed at addressing the challenges facing health personnel working in rural areas in KwaZulu-Natal, South Africa. Afr J Prim Health Care Fam Med 2017;9:e1-e8.

13. le Roux KW, Couper I. Rural district hospitals-Essential cogs in the district health system-And primary healthcare re-engineering. S Afr Med J 2015;105:440-441.

14. Plazy M, Perriat D, Gumede D, et al. Implementing universal HIV treatment in a high HIV prevalence and rural South African setting-Field experiences and recommendations of health care providers. PLoS One 2017;12:e0186883.

15. Callaghan M, Ford N, Schneider H. A systematic review of task-shifting for HIV treatment and care in Africa. Hum Resour Health 2010;8:8.

16. Rikhotso SR, Williams MJ, De Wet G. Student nurses' perceptions of guidance and support in rural hospitals. Curationis 2014;37:e1-e6.

17. Chimbindi N, Bor J, Newell ML, et al. Time and money: The true costs of health care utilization for patients receiving "free" HIV/TB care and treatment in rural KwaZuluNatal. J Acquir Immune Defic Syndr 2015;70:e52.

18. South African National Department of Health. Tried and Tested: Models for the Scale Up of HIV Prevention, Treatment and Care from South Africa and Beyond. 2010. 
Available at: www.ihi.org/resources/Documents/Triedand Tested_ModelsforScaleUpHIVPrevention_Book.pdf

19. Rural Health Advocacy Project. Rural Health Fact Sheet 2015. Available at: http://rhap.org.za/wp-content/uploads/ 2015/09/RHAP-Rural-Health-Fact-Sheet-2015-web.pdf (Last accessed March 15, 2019).

20. Baleta A. Rural hospital beats the odds in South Africa. Lancet 2009;374:771-772.

21. Statistics South Africa. Census 2011. Available at: https:// wazimap.co.za (Last accessed March 11, 2019).

22. Goudge J, Gilson L, Russell S, et al. The household costs of health care in rural South Africa with free public primary care and hospital exemptions for the poor. Trop Med Int Health 2009;14:458-467.

23. Bateman C. Drug stock-outs: Inept supply-chain management and corruption. S Afr Med J 2013;103:600-602.

24. Department of Health Republic of South Africa. The South African Antiretroviral Treatment Guidelines 2013. 2013. Available at: www.sahivsoc.org/Files/2013\%20ART\%20 Treatment $\% 20$ Guidelines\%20Final\%2025\%20March\%20 2013\%20corrected.pdf (Last accessed March 11, 2019).

25. National Department of Health. Adherence guidelines for HIV, TB, and NCDs. 2016. Available at: www.nacosa.org .za/wp-content/uploads/2016/11/Integrated-AdherenceGuidelines-NDOH.pdf (Last accessed March 15, 2019).

26. Kaposhi BM, Mqoqi N, Schopflocher D. Evaluation of antiretroviral treatment programme monitoring in Eastern Cape, South Africa. Health Policy Plan 2015;30:547-554.

27. Maughan-Brown B, Smith P, Kuo C, et al. A conditional economic incentive fails to improve linkage to care and antiretroviral therapy initiation among HIV-positive adults in Cape Town, South Africa. AIDS Patient Care STDS 2018;32:70-78.

28. Penn AW, Azman H, Horvath H, et al. Supportive interventions to improve retention on ART in people with HIV in low-and middle-income countries: A systematic review. PLoS One 2018;13:e0208814.

29. Omonaiye O, Nicholson P, Kusljic S, Manias E. A metaanalysis of effectiveness of interventions to improve ad- herence in pregnant women receiving antiretroviral therapy in sub-Saharan Africa. Int J Infect Dis 2018;74:71-82.

30. Bemelmans M, Baert S, Goemaere E, et al. Communitysupported models of care for people on HIV treatment in sub-Saharan Africa. Trop Med Int Health 2014;19:968977.

31. South African National AIDS Council Trust. South Africa Global AIDS Response Progress Report (GARPR). 2015. Available at: www.sanac.org.za/download/563/.../garpr_reporthigh-res-for-print-june-15-2016.pdf (Last accessed March 15, 2019).

32. Omole O, Semenya M. Treatment outcomes in a rural HIV clinic in South Africa: Implications for health care. South Afr J HIV Med 2016;17:1-6.

33. Boyles TH, Wilkinson LS, Leisegang R, et al. Factors influencing retention in care after starting antiretroviral therapy in a rural South African programme. PLoS One 2011;6:e19201.

34. Houlihan CF, Bland RM, Mutevedzi PC, et al. Cohort profile: Hlabisa HIV treatment and care programme. Int $\mathbf{J}$ Epidemiol 2011;40:318-326.

35. Mutevedzi PC, Lessells RJ, Heller T, et al. Scale-up of a decentralized HIV treatment programme in rural KwaZuluNatal, South Africa: Does rapid expansion affect patient outcomes? Bull World Health Organ 2010;88:593-600.

36. Tweya H, Feldacker C, Estill J, et al. Are they really lost? "True" status and reasons for treatment discontinuation among HIV infected patients on antiretroviral therapy considered lost to follow up in Urban Malawi. PLoS One 2013;8:e75761.

Address correspondence to: Mary Jane Rotheram-Borus, PhD

Department of Psychiatry

University of California, Los Angeles 10920 Wilshire Blvd. Suite 350 Los Angeles, CA 90024

E-mail: cchpublications@mednet.ucla.edu 